\title{
'You cannot expect miracles to happen overnight': patience pays off when you wish to establish a new adrenocortical carcinoma cell line
}

\author{
Enzo Lalli $(\mathbb{D}$
}

Institut de Pharmacologie Moléculaire et Cellulaire CNRS UMR7275, Université Côte d'Azur, Inserm, Valbonne, France

Correspondence should be addressed to E Lalli

Email

ninino@ipmc.cnrs.fr

\begin{abstract}
Growing attention is being paid to the association of adrenocortical carcinoma (ACC), a rare endocrine malignancy, to cancer predisposition syndromes caused by germline mutations in genes involved in the control of genome stability. Tumour cells with a defective DNA mismatch repair pathway have a high mutation burden, which results in the production of tumour-associated specific neoantigens and in an increase of the sensitivity to therapies that loosen the constraints of tumour attack by the immune system. The study by Landwehr et al. published in a recent issue of the European Journal of Endocrinology describes a patient with an aggressive ACC bearing a germline MUTYH mutation with loss of heterozygosity in the tumour and accumulation of 8-hydroxyguanine in its genomic DNA. The authors managed to establish a novel differentiated cell line from that tumour which bears the stigma of the defective DNA repair mechanism in its genome. The availability of this new cell model inside the expanding toolbox of the ACC cell lines will allow for novel experimental possibilities, in particular for the study of the tumour microenvironment and the response to immunotherapy.
\end{abstract}

Adrenocortical carcinoma (ACC) is a rare endocrine malignancy whose medical therapy relies on the use of mitotane, a drug with limited efficacy and potential toxicity. Combination chemotherapy (etoposide-doxorubicincisplatin plus mitotane) is used in patients with advanced disease and is able to improve progression-free but not overall survival. Genomic studies have evidenced the driver mutations in sporadic ACC and also revealed that ACC could be associated with cancer predisposition syndromes in patients bearing germline mutations in TP53 (Li-Fraumeni syndrome) or in genes involved in DNA mismatch repair (MMR) (Lynch syndrome) $(1,2)$. This is particularly important in the clinic, since tumours with defects in the DNA repair pathway have a high mutational burden and production of tumour-associated specific neoantigens which may render them sensitive to immunotherapy.

Cell models that recapitulate the entire spectrum of ACC heterogeneity, phenotypic aggressiveness and sensitivity to mitotane and other drugs are lacking. The overwhelming majority of studies focused on the 'historic' NCI-H295 (or its H295R subclone growing attached, developed in Dr Rainey'slaboratory), theonly differentiated human ACC cell line available until very recently. This is a serious drawback for the research in the field of ACC compared to most other cancer types. Incidentally, many studies also used the SW13 cell line, alleged to be of adrenocortical origin. However, this cell line, derived from an adrenal metastasis of a small cell carcinoma, does not express adrenocortical differentiation markers and is 
not steroidogenic, so it cannot be considered as a valid in vitro model for ACC. Efforts to establish additional ACC cell lines have been made in many laboratories but with no success. However, the good news is that a few new bona fide ACC cell lines have recently been established, which were originally derived as patient-derived xenografts (PDX) in nude mice and then adapted to grow in culture (3). These new ACC cell lines differ in their pattern of genomic alterations, expression of adrenocortical markers, steroidogenic capacities and sensitivity to mitotane and other chemotherapeutic drugs.

The results published by Landwehr et al. in a recent issue of the European Journal of Endocrinology (4) represent a new important step forward in the process of increasing the diversity of the cell line models available for ACC research. The authors established a new cell line (JIL2266) from the tumour mass resected from a female patient with severe Cushing's syndrome bearing a large, high-grade ACC associated with metastases in the lung. The patience showed by the authors in taking care of their primary cultures has to be particularly commended since they report that the cells were first passaged 6 months after tumour dissociation and establishment of the culture! Slow proliferation ensued until a point where cell doubling time dramatically decreased, and the cells started to grow continuously. It is interesting to remark that JIL-2266 did not need a previous passage as xenograft in nude mice before culture in the dish nor the presence of feeder cells, at variance with the other recently established ACC cell lines. Probably the gentle dissociation method and the use of special plasticware and of Y-27632, a Rho-kinase (ROCK) inhibitor, in the culture medium all had an important role to promote cell attachment and survival in the early phases of the in vitro culture. The adrenocortical origin of the JIL-2266 cells was confirmed by the positivity of SF-1 immunostaining. Production of small amounts of progesterone, 17-OH-progesterone, DHEA, androstenedione and testosterone was detected by LC-MS/MS. Lower steroid secretion was associated with higher cell line passages, probably because of the accumulation of mutations negatively affecting the cells' steroidogenic capacity. While these data are in contrast with the clinical presentation of the patient, they may be explained by the high tumour burden which caused symptoms by steroid hormone excess even in the presence of inefficient tumour cell steroidogenesis. JIL2266 cells are significantly less sensitive to mitotane than NCI-H295R cells. Furthermore, the authors characterised the pattern of genomic alterations in the tumour tissue and in the JIL-2266 cell line by whole exome sequencing, identifying a few hundred somatic variants, most of which were shared between the primary tumour and the cell line. Remarkably, a hemizygous TP53 stop mutation was present in both of them, as well as a loss of heterozygosity (LOH) for a germline mutation (p.R106W) in the MUTYH gene encoding a protein involved in DNA glycosylaseinitiating base-excision repair. It catalyses the removal of adenine mispaired with 8-hydroxyguanine (8OHG), an oxidised form of guanine produced by oxidative damage, from dsDNA. The p.R106W mutation was detected in $0.01 \%$ of the AACR GENIE cancer cases and was previously shown to impair the DNA glycosylase activity of the protein (5). Biallelic germline inactivating MUTYH mutations predispose to MUTYH-associated polyposis (MAP), characterised by multiple colorectal polyps and carcinomas. Tumours bearing MUTYH mutations display a specific pattern of genomic alterations, characterised by the accumulation of $\mathrm{C}>\mathrm{A}$ transversions, due to incorrect replication of $8 \mathrm{OHG}$ (signature 18 in the Catalogue of Somatic Mutations in Cancer - COSMIC database). MUTYH mutations with $\mathrm{LOH}$ were previously identified in ACC cases from the ENSAT and TCGA studies displaying genomic alterations consistent with signature 18 (6). A signature 18 pattern was also present in the ACC described by Landwehr et al. and in the JIL-2266 cell line. The patient presented a positive family history for cancer, with a cerebellar tumour in the mother and breast cancer in the sister and grandmother. In addition, immunostaining for $8 \mathrm{OHG}$ showed strong positivity both in the primary tumour and in the JIL-2266 cell line, while another ACC without MUTYH alterations was completely negative. These data definitively confirm the pathogenetic role of the p.R106W MUTYH mutation in this patient with ACC.

The case described by Landwehr et al. underlines the importance of germline mutations in the pathogenesis of ACC, which induces genomic instability and high tumour mutational burden. According to an interesting communication on a large cohort of ACC cases presented at ENDO 2021, up to $13 \%$ of them may harbour alterations in the MMR pathway (7), which brings forward the option to treat those patients with immunotherapy. The availability of JIL-2266 cells inside the expanding toolbox of the ACC cell lines will allow for novel experimental possibilities, in particular for the study of the tumour microenvironment and the response to immunotherapy in humanised mouse models, similar to what has already been performed for the Lynch syndrome ACC cell line CU-ACC2 (8). 


\section{Declaration of interest}

The author declares that there is no conflict of interest that could be perceived as prejudicing the impartiality of this commentary.

\section{Funding}

This commentary article did not receive any specific grant from any funding agency in the public, commercial or not-for-profit sector.

\section{References}

1 Assié G, Letouzé E, Fassnacht M, Jouinot A, Luscap W, Barreau O, Omeiri H, Rodriguez S, Perlemoine K, René-Corail F et al. Integrated genomic characterization of adrenocortical carcinoma. Nature Genetics 201446 607-612. (https://doi.org/10.1038/ng.2953)

2 Zheng S, Cherniack AD, Dewal N, Moffitt RA, Danilova L, Murray BA, Lerario AM, Else T, Knijnenburg TA, Ciriello G et al. Comprehensive pan-genomic characterization of adrenocortical carcinoma. Cancer Cell 201629 723-736. (https://doi.org/10.1016/j.ccell.2016.04.002)

3 Pinto EM, Kiseljak-Vassiliades K \& Hantel C. Contemporary preclinical human models of adrenocortical carcinoma. Current Opinion in Endocrine and Metabolic Research 20198 139-144. (https://doi.
4 Landwehr LS, Schreiner J, Appenzeller S, Kircher S, Herterich S, Sbiera S, Fassnacht M, Kroiss M \& Weigand I. A novel patient-derived cell line of adrenocortical carcinoma shows a pathogenic role of germline MUTYH mutation and high tumour mutational burden. European Journal of Endocrinology 2021184 823-835. (https://doi. org/10.1530/EJE-20-1423)

5 Shinmura K, Goto M, Tao H, Kato H, Suzuki R, Nakamura S, Matsuda T, Yin G, Morita M, Kono S et al. Impaired 8-hydroxyguanine repair activity of MUTYH variant p.Arg109Trp found in a Japanese patient with early-onset colorectal cancer. Oxidative Medicine and Cellular Longevity 20142014 617351. (https://doi.org/10.1155/2014/617351)

6 Pilati C, Shinde J, Alexandrov LB, Assié G, André T, Hélias-Rodzewicz Z, Ducoudray R, Le Corre D, Zucman-Rossi J, Emile JF et al. Mutational signature analysis identifies MUTYH deficiency in colorectal cancers and adrenocortical carcinomas. Journal of Pathology 2017242 10-15. (https://doi.org/10.1002/path.4880)

7 Pozdeyev N, Fishbein LM, Gay LM, Sokol ES, Hartmaier R, Ross JS, Darabi S, Demeure MJ, Kar A, Foust LJ et al. Insights from targeted genetic analysis of 364 adrenocortical carcinomas. Journal of the Endocrine Society 20215 A168. (doi: 10.1210/jendso/bvab048.339)

8 Lang J, Capasso A, Jordan KR, French JD, Kar A, Bagby SM, Barbee J, Yacob BW, Head LS, Tompkins KD et al. Development of an adrenocortical cancer humanized mouse model to characterize anti-PD1 effects on tumor microenvironment. Journal of Clinical Endocrinology and Metabolism 2020105 26-42. (https://doi. org/10.1210/clinem/dgz014)

Received 15 April 2021

Accepted 7 June 2021 